\title{
Multidisciplinary care of craniosynostosis
}

This article was published in the following Dove Press journal:

Journal of Multidisciplinary Healthcare

6 July 2017

Number of times this article has been viewed

\section{Edward P Buchanan' \\ Yunfeng Xue' \\ Amy S Xue' \\ Asaf Olshinka' \\ Sandi Lam²}

'Michael E. DeBakey Department of Surgery, Division of Plastic Surgery, ${ }^{2}$ Michael E. DeBakey Department of Surgery, Division of Neurosurgery, Baylor College of Medicine, Houston, TX, USA
Correspondence: Edward P Buchanan Michael E. DeBakey Department of Surgery, Division of Plastic Surgery, Baylor College of Medicine, 670I Fannin St, CC 610.00, Houston, TX 77030, USA

$\mathrm{Tel}+\mathrm{I} 8328223190$

$\mathrm{Fax}+\mid 8328253192$

Email ebuchana@bcm.edu
Abstract: The management of craniosynostosis, especially in the setting of craniofacial syndromes, is ideally done in a multidisciplinary clinic with a team focused toward comprehensive care. Craniosynostosis is a congenital disorder of the cranium, caused by the premature fusion of one or more cranial sutures. This fusion results in abnormal cranial growth due to the inability of the involved sutures to accommodate the growing brain. Skull growth occurs only at the patent sutures, resulting in an abnormal head shape. If cranial growth is severely restricted, as seen in multisuture craniosynostosis, elevation in intracranial pressure can occur. Whereas most patients treated in a multidisciplinary craniofacial clinic have non-syndromic or isolated craniosynostosis, the most challenging patients are those with syndromic craniosynostosis. The purpose of this article was to discuss the multidisciplinary team care required to treat both syndromic and non-syndromic craniosynostosis.

Keywords: multidisciplinary team care, syndromic craniosynostosis, nonsyndromic craniosynostosis

\section{Introduction \\ Epidemiology and genetics}

The prevalence of craniosynostosis ranges from 3.1 to 5.06 per 10,000 births. $^{1-3}$ In a recent study, $84 \%$ of patients presented with isolated craniosynostosis, $7 \%$ with additional clinical symptoms, and $9 \%$ with suspected syndromic craniosynostosis. ${ }^{3}$ Single suture craniosynostosis is more common than multisuture craniosynostosis. The most common type is sagittal craniosynostosis, accounting for $40 \%-60 \%$ of all cases. $^{2-5}$ In comparison, bicoronal craniosynostosis is the most common presentation in patients with multisuture craniosynostosis ${ }^{6}$ and can present in either syndromic or non-syndromic patients. Craniosynostosis has been described in more than 150 different syndromes. ${ }^{7,8}$

Fibroblast growth factor receptor (FGFR) mutations are most frequently cited in association with craniosynostosis, especially syndromic types. The mutation usually leads to a gain-of-function genetic defect that is autosomal dominant in inheritance. ${ }^{9}$ Some notable craniofacial (CF) syndromes with FGFR mutations include Apert, Crouzon, Pfeiffer, and Muenke. Other syndromes, such as Saethre-Chotzen and Carpenter, are linked to non-FGFR mutations. Although significant advances have been made in molecular genetics research, a single genetic anomaly has not been identified as a cause for craniosynostosis. 


\section{Multidisciplinary team}

Multidisciplinary CF teams commonly assume the responsibility of treating patients with craniosynostosis. These teams consist of multiple different specialists, each with their own expertise, but all with the common goal of comprehensive and thorough care (Table 1). Most CF teams are championed by a team coordinator who functions as the liaison between the patient and the treating team. This individual gives the family a common point of contact to coordinate all care needs. Even though the CF coordinator at our institution is a nurse with experience in CF conditions, any medical professional with care and dedication to this patient population can function as a coordinator. This individual is one of the most important parts of any team-patient (family) relationship because they are responsible for all aspects of team coordination.

As listed here, CF teams consist of many members with varying expertise related to the care of a patient with a CF condition. To begin with, CF pediatrician or nurse practitioner serves as the primary care provider in the team and is one of the key members. They have special expertise in the specific needs of patients with CF conditions and understand the way their symptoms affect activities of daily living. They can help counsel families on what to expect as their child grows and the challenges they will face as they become more social and interactive with others. Their experience with patients and families who suffer from CF conditions helps them provide information and comprehensive care as only a primary health care provider can do. They are an essential component of the team and are

Table I List of craniofacial team members

Craniofacial team members
Audiologists
Dentist/orthodontists
Otolaryngologists
Ophthalmologists
Geneticists and genetic counselors
Neurosurgeons
Nurse practitioners
Nurses
Nutritionists
Occupational/physical therapists
Oral and maxillofacial surgeons
Pediatricians
Plastic and reconstructive surgeons
Psychologists
Researchers
Respiratory care specialists
Social workers
Speech and language pathologists
Support staff

highly valued to make each family and patient understand their care and prognosis.

Audiologists are important members of the team because many children with craniosynostosis and CF conditions can have ear and hearing issues. If hearing is untested and undertreated, further problems with speech and language development can occur. These issues can also lead to significant impacts on emotional and social development that are absolutely necessary for learning. The audiologist on each team will screen patients entering the clinic for hearing difficulties to identify any issues for further treatment. This allows the team to intervene with appropriate care if hearing issues are present.

Dentists and orthodontists are absolutely essential to any complete CF team due to the frequency of dental and dentofacial deformities in children who have syndromic craniosynostosis. Whereas including a dentist early in life is important for any person, patients with syndromic craniosynostosis have a particular threat to achieving optimal oral hygiene. Preservation of teeth and appropriate dental care are important. Pediatric dentists are involved in patient care early to address healthy oral hygiene and routine maintenance and cleanings. CF orthodontists usually get involved in the care of these patients later after growth and eruption of the permanent teeth. Midface hypoplasia is one of the most common dentofacial problems patients with syndromic craniosynostosis present with. Depending on the associated symptoms and indications, correction of this problem can occur early in life, as in the first 5 years, or later, when the CF skeleton is more mature. The CF orthodontists assist the treating surgeons by helping to plan midface surgeries to achieve the most ideal postoperative results. Further, after patients reach skeletal maturity, CF orthodontists will assist with the planning of orthognathic surgery to achieve a normal occlusive relationship and correct any residual dentofacial deformities. Preoperative planning and postoperative orthodontic follow-up is key to successful long-term outcomes.

Otolaryngologists are essential members of the CF team, especially as it relates to the treatment of syndromic craniosynostosis. Syndromic patients are not only affected by dentofacial deformities that involve the upper airway, but they also suffer from primary airway pathologies. Early airway evaluation and treatment is highly recommended to avoid future airway emergencies. Midface hypoplasia can cause a constriction of the nasal airway and difficult breathing through the nose and mouth. Pathology in other parts of the airway can have a compounding impact on regular breathing and sleeping. Common diagnoses include 
laryngomalacia, tracheomalacia, and tracheacartilagenous sleeves. Otolaryngologists are further needed for the thorough evaluation of the auditory system and to collaborate with the audiology team given the high prevalence of hearing issues in this population. Tracheostomies are a common occurrence in patients with syndromic craniosynostosis given the multiple levels of airway pathology. Tracheostomy maintenance is essential to prevent longterm complications.

Ophthalmologists are necessary to any $\mathrm{CF}$ team given the number of eye-related issues. The orbital deformities created by craniosynostosis are common and can affect the normal functioning of the eye. They require the expertise of ophthalmologists to assist with correction. Further, craniosynostosis can cause an elevation in intracranial pressure due to the limited space caused by fused cranial sutures. If this is present, ophthalmologists can often identify it during a routine eye exam. Often times, this is the only way to detect elevated intracranial pressure without doing more invasive testing. Most craniosynostosis patients, especially the syndromic ones, are followed and evaluated by an ophthalmologist on a yearly basis to assess the optic nerve for evidence of elevated intracranial pressure.

Geneticists and genetic counselors provide patients and their families with answers to questions related to their diagnosis, prognosis, and potential for inheritance. Having a child born with any medical condition, either related to a syndrome or not, is a concerning and anxiety ridden time for any family. Proper consultation and evaluation by a geneticist and genetic counselor are important to help families understand and cope with the diagnosis. Questions regarding future children, possible inheritance, and prognosis can be addressed by a genetic specialist to ensure a thorough understanding of the condition and preparation for future care.

Pediatric neurosurgeons are necessary to help with the surgical treatment of patients with craniosynostosis. Due to the involvement of the cranial bones and the resulting head shape changes, operations to treat craniosynostosis involve reshaping the skull to allow for cranial growth. These surgeries are done with a neurosurgeon as well as a CF trained plastic surgeon. Syndromic craniosynostosis patients often suffer from other neurosurgical diseases, such as hydrocephalus and Arnold-Chiari malformation. Frequent evaluations by a pediatric neurosurgeon are essential in avoiding future issues related to the neurologic development.

The dentofacial and neurologic issues patients with craniosynostosis suffer from can leave them malnourished and in need of extra care as it relates their nutrition. A properly trained nutritionist who understands the unique challenges of these patients maintain normal growth and development.

Occupational and physical therapists can help families and patients with mobility and coordination issues that can result due to some of the neurologic problems that can arise. Failure to advance with peers in regards to coordination and motor tasks can be improved with the constant help of physical and occupational therapists. Further, after surgery, many children can be extremely weak and be in need of services to help with recovery. The proper amount of therapy can help children return to their preoperative state and shorten postoperative recovery periods.

Oral and maxillofacial surgeons are commonly a part of CF teams that help to surgically treat dentofacial deformities. Syndromic patients often present with severe midface hypoplasia and will need correction as skeletal maturity is reached. Even if a midface operation has been completed at an earlier age, malocclusion can still be present and needs to be corrected to help with eating, speech problems, and appearance. A thorough evaluation by an oral surgeon in combination with a $\mathrm{CF}$ orthodontist needs to be done at skeletal maturity to ensure that all dentofacial deformities are corrected.

Plastic and reconstructive surgeons, with specific training in $\mathrm{CF}$ surgery, provide patients with the surgical care path required for their diagnosis. Whether a single surgery is required at an early age or multiple surgeries as children grow, a CF surgeon has knowledge related to all symptoms patients with craniosynostosis can experience. The CF surgeon is thoroughly aware of the natural history of all associated conditions and can map out the potential issues that may develop. Preventive surgery is preferred and should be instituted at the appropriate age so that repeat operations can be avoided. Issues related to skull growth, orbits, airways, midface, mandible, and dentofacial deformities are all considered by the CF surgeon.

Psychologists are required to assess the neuropsychological growth and development of patients. There is much debate regarding the long-term cognitive and psychological development of patients suffering from craniosynostosis. Many of these effects can only be measured by experienced psychologists who understand this condition and its implications for development. Research in this field is active and ongoing, stressing the importance of psychologists as active members of the team. A strong psychology team can help every other team member with 
some of the most important, yet underappreciated, parts of care.

Researchers should be included in all CF teams to track patients from birth until eventual discharge. There is much about the growth and development of craniosynostosis patients that we do not know or understand. Issues related to morphology, psychology, emotional adjustment, and long-term growth are not very well understood, leaving the craniosynostosis field fertile for potential research opportunities. These shortcomings make it difficult to fully develop an outcome-based care pathway. When research specially focuses on all aspects of craniosynostosis, these pathways can be implemented, and the best possible care can be delivered to our patients.

Respiratory therapists are important for patients with craniosynostosis who suffer from airway issues, such as sleep apnea, midface hypoplasia, tracheocartilagenous sleeves, and tracheostomies. Whereas their importance is most apparent during the perioperative periods, maintenance of healthy airway protocols is important to prevent unnecessary hospitalizations. Avoidance of respiratory and pulmonary complications helps families remain out of the hospital and allows for timely surgeries to prevent future complications.

Social workers help families and patients obtain the services they need as they go through their treatment. The medical and surgical care these patients require is extensive and can be overwhelming, physically, emotionally, and mentally. Social workers can provide families with relief from some of these burdens and can help track the course of care. They also help families with other services that may make living with a child with specialized care easier. A constant line of communication is necessary to help these families receive the care they need and ensure that their child is getting appropriate resources.

Speech and language pathologists help the children very early in life with any feeding difficulties and later with speech delays or issues. Due to neurologic and dentofacial deformities, many of these children may experience delays in speech production and language skills. Constant and continual evaluation from speech therapists will help the team identify issues early and institute therapies that can help with future growth and development.

The support staff on any multidisciplinary team is helpful with the logistical side of care. These individuals are usually the glue that keeps the team together, coordinated, and allow for the seamless flow of patients through the clinic. Coordination of patient visits, surgery, and follow-ups is a thankless, tireless, and underappreciate part of any team. If coordination is not done, families will feel as if the team is disorganized. If the confidence in the team diminishes, it can subsequently affect follow-up visits and future team interactions. It is important that the support staff is aware of the unique and challenging opportunity that they are a part of and the importance of a well-functioning multidisciplinary team.

\section{Non-syndromic craniosynostosis}

The most commonly encountered forms of non-syndromic craniosynostosis include sagittal, unicoronal, bicoronal, metopic, and lambdoid craniosynostosis (Figure 1). Sagittal craniosynostosis is the most common type and involves fusion of the sagittal cranial suture. It presents with an expanded anteroposterior dimension, pterion constriction, occipital bullet, and frontal bossing. The overall deformity is referred to as scaphocephaly or a "boat-shaped" skull.

Metopic craniosynostosis involves fusion of the metopic cranial suture and has seen an increase in prevalence in recent years. It is estimated to account for approximately $20 \%$ of craniosynostosis cases. ${ }^{3,11,12}$ In metopic craniosynostosis, the forehead presents with an overall triangular appearance, referred to as trigonocephaly. Other clinical findings include keel-shaped forehead, hypotelorism, and pterion constriction.

Plagiocephaly, from the Greek root plagio - meaning sideways or slanting, is a general term describing any asymmetry of the skull in the coronal plane. This condition can occur in either the front or back, as seen in unilateral coronal craniosynostosis, unilateral lambdoid craniosynostosis, or deformational plagiocephaly. Unilateral coronal craniosynostosis, due to premature fusion of one coronal suture, results in ipsilateral frontal bone flattening, elevation of the ipsilateral superior orbital rim, and a relative contralateral frontal bossing. Bilateral coronal craniosynostosis occurs when both of the coronal sutures fuse prematurely. Whereas it is commonly seen in patients with syndromic craniosynostosis, it can occur as an isolated finding. ${ }^{13}$ The premature fusion of both coronal sutures results in brachycephaly, limited anteroposterior skull growth, and compensatory growth in transverse skull width.

Lambdoid craniosynostosis, the least common, involves the premature fusion of the lambdoid suture in the posterior skull and will result in a posterior plagiocephaly. The affected suture will cause an ipsilateral skull flattening and a prominent mastoid bulge. The contralateral parietal bone will be more prominent to allow for compensatory growth.

Lambdoid craniosynostosis can be confused with another condition causing a posterior skull flattening, deformational 


\section{Types of Craniosynostosis}

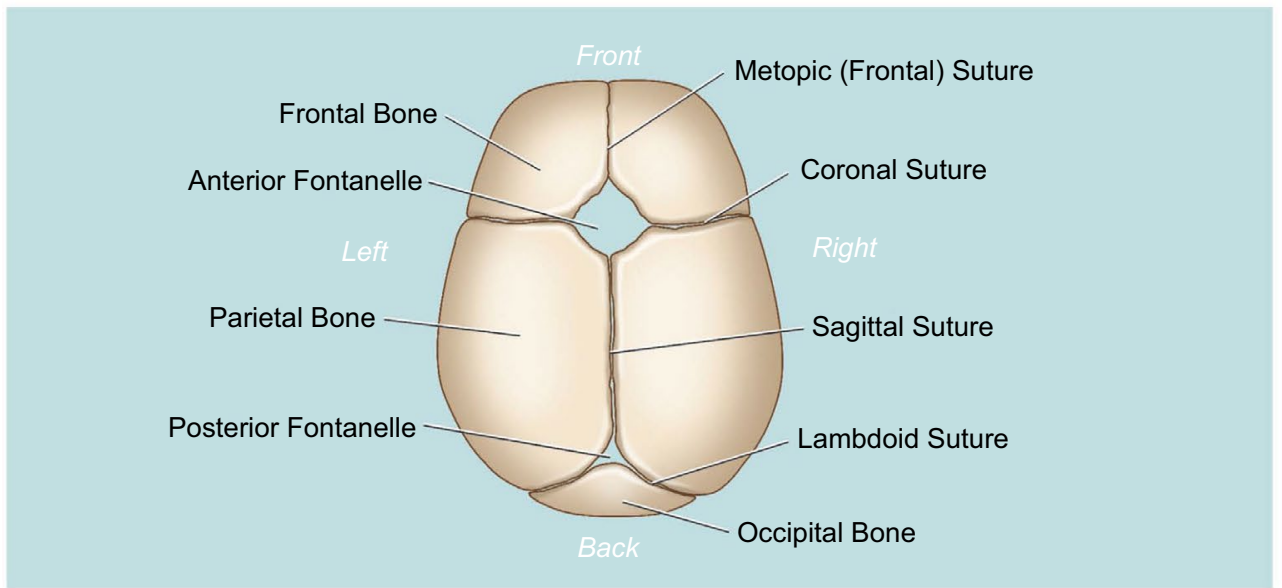

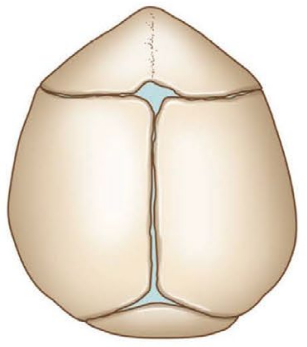

Metopic

Synostotic Trigonocephaly

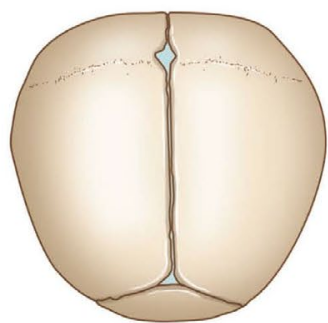

Bicoronal

Synostotic Brachycephaly

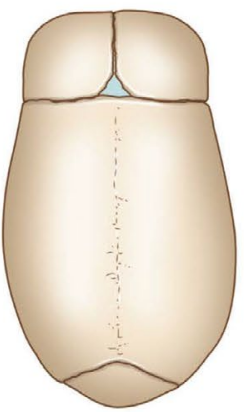

Sagittal

Synostotic Scaphocephaly

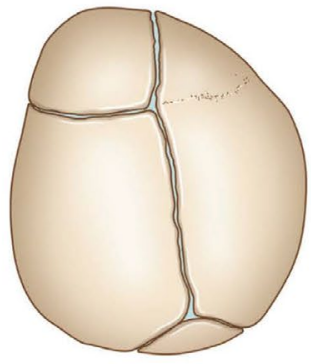

Unicoronal

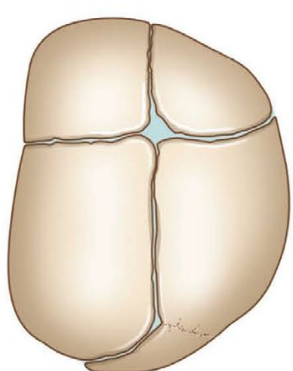

Lambdoid

Synostotic Posterior Plagiocephaly

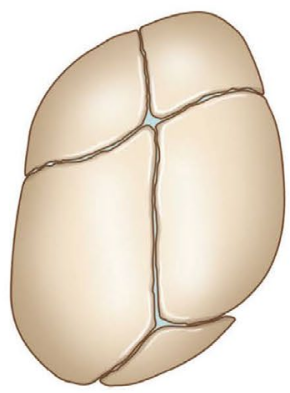

(All Sutures Open)

Synostotic Anterior Plagiocephaly Deformational Posterior Plagiocephaly

Figure I Types of craniosynostosis.

plagiocephaly. This skull flattening is not related to premature suture fusion, but rather to the molding of the soft infant cranium due to pressure over a period of time. It does not require surgical correction because further skull growth will correct the flatness. Deformational plagiocephaly can be corrected with helmet therapy if identified before the first year of life. Whereas helmet therapy works actively by guiding the direction of skull growth and correct the flatness more quickly, much of the correction will occur with normal growth without the helmet.

Most cases of non-syndromic craniosynostosis are initially discovered by family members or the treating pediatrician early in life. Referral to CF clinic for evaluation is the first step in the treatment pathway. Many clinics will obtain a radiographic image of the skull to confirm the diagnosis. The preferred imaging modality for our clinic is computed 
axial tomographic (CT) with three-dimensional (3D) rendering. This scan is done with a low-dose radiation protocol to limit the amount of radiation the child receives during the study. Due to this concern, some centers will forgo the CT scan and proceed with a cranial ultrasound to evaluate the patency of the cranial sutures. Whereas this method is effective to determine the extent of cranial suture involvement, it gives little information on the entirety of the 3D deformity, involvement of other cranial sutures, and evaluation of intracranial contents. Possible intracranial abnormalities include Arnold-Chiari malformations, hydrocephalus, and brain malformations. The presence of these findings may necessitate early neurosurgical intervention, prior to any cranial vault remodeling, although this is more commonly seen in syndromic patients.

During the initial evaluation, the family and patient briefly meet with or they get introduced to all team members. This initial visit, although comprehensive, allows the family to understand the breadth of care their child will need and provides them with information regarding the condition. Thus, an initial relationship between the family and the CF coordinator is established. Verbal and written education is provided regarding the diagnosis, prognosis, required operations, contact points, and future appointments. All questions are answered, and families are encouraged to call or e-mail with further inquiries, concerns, and questions. This initial visit is probably the most important interaction between the family and their team. It sets the tone for future care and has the ability to instill confidence about the team. Some members of the team will spend more time during this initial visit than others, which is highly dependent on where the child is in their care spectrum.

Clinic visits can be broken down into initial consultation, preoperative visit, postoperative visits, and routine followups. As mentioned previously, the initial consultation is the most important visit because it introduces the patient and families to the team members and begins the relationship that will form the basis for all future clinical encounters. Most, if not all members of the team, should meet the patients and family members, introduce themselves, and explain their roles as members of the team. Information, both verbal and written, is provided to patients about their condition. A treatment timeline is provided and explained depending on individual diagnosis. Families should leave this visit with appropriate information about their team and the care their child needs moving forward.

During the preoperative clinic visit, families will meet with the involved surgical teams for a thorough and com- plete explanation of the surgical intervention they will receive. The two most common teams they will meet with are the neurosurgery and plastic surgery teams. Further, a pre-anesthesia clinic visit by a pediatric anesthesiologist with specific experience with CF conditions will take place. Preoperative labs, including a cell blood count (CBC) and coagulation levels as well as a type and cross matching is commonly ordered. Families will also meet with the CF nurse to discuss all perioperative issues. These include length of hospital stay, what to expect after surgery in both the short and long terms, wound care, postoperative instructions, and follow-up appointments.

Postoperative clinic visits and routine follow-up visits ensure that the child is recovering from surgery as expected and meeting developmental milestones. Yearly ophthalmologic exams are performed for each child to evaluate the optic nerve and retina for signs of elevated intracranial pressure. Further, patients are questioned regarding headaches, photophobia, or unexplained nausea and vomiting. Neuropsychological testing is only ordered and performed if family members are concerned with their child's neurological development. Patients are examined for skull shape and presence of any persistent cranial defects. Skull shape is evaluated and measured in various different ways. First, head circumference is measured to assess normal skull size and growth patterns. A cranial index is performed, comparing the skull width to skull length, to assess normal ratios. 3D photography is currently being used to evaluate skull shape and compare it to normal age-matched patients. Whereas the technology to capture 3D image is common, software to measure these shape changes pre- and postoperatively is not currently available. Once 3D changes can be measured consistently on the same patient in the pre- and postoperative settings, a better understanding of the effectiveness of our surgical techniques can be understood. Patients are normally followed on a yearly basis until early adulthood when they are discharged from the clinic. They are instructed to return if any issues occur which they believe are related to their diagnosis.

Surgical planning depends on the involved sutures. Age of first surgery is dependent on diagnosis and can range from as early as 2-3 months to as late as 10-12 months, depending on the treating institution. For patients requiring a frontal correction, involving the forehead and superior orbital bar, the surgery is usually delayed until 10-12 months of age. Patients with unicoronal, bicoronal, and metopic craniosynostosis usually fall into this category. This time is selected because of the belief that the bone is strong enough at this age to withstand a rigid reconstruction without future 
relapse. Sagittal suture craniosynostosis is commonly treated at 6-9 months of age, to take advantage of the rapid skull growth as well as soft cranial bones, thereby allowing more facile bone shaping. Metopic, sagittal, and unilateral lambdoids are currently being treated at an even earlier age with an endoscopic technique. An endoscopic technique is usually performed as early as $2-3$ months of age. Strip craniectomies of the involved suture combined with barrel stave osteotomies of the deformities are performed. Patients are placed in a molding helmet postoperatively to help achieve the desired shape. Helmets are required until the proper shape is achieved. Patients who undergo open operations usually do not require a course of postoperative helmet therapy.

\section{Syndromic craniosynostosis}

The most common syndromes that are encountered with craniosynostosis include Crouzon, Apert, Pfeiffer, Muenke, and Saethre Chotzen syndromes. Although there are a variety of other syndromes that can present with craniosynostosis, these are some of the most common that present in a $\mathrm{CF}$ clinic. Given their complexity, these patients should be treated from an early age by a multidisciplinary CF team. Team participation will allow for an analysis of all medical issues and help with prioritization of care. The exact care for each syndrome is beyond the breadth of this chapter; however, important aspects of syndromic patients are discussed here.

Syndromic craniosynostosis patients frequently present with multisuture cranial involvement, but single-suture involvement can also be seen. Genetic testing should be done on all patients treated for craniosynostosis since a syndromic diagnosis usually requires a more extensive level of treatment. Neurosurgical evaluation for the presence of any associated neurological abnormalities, Chiari malformation or hydrocephalus, should be done early. Premature suture fusion in the cranial base is often found with syndromic patients and can affect the growth of the midface as well as the patency of the draining cerebral veins. Midface hypoplasia is a common finding in syndromic patients, and depending on the severity, the state of the airway, the need for orbital globe protection, an early midface operation may be indicated. Each syndrome has its own unique pattern of multisuture involvement, and the resulting deformities are evident on clinical presentation. Skull expansion should be considered in accordance to the syndrome being treated and the sutures involved. Surgical intervention for intracranial volume restriction usually occurs within the first 2 years of life.

Early ophthalmologic involvement should be obtained to assess for ocular abnormalities and increased intracranial pressure. The extent of midface hypoplasia predicts the need for an early or late midface surgical intervention. Malignant exophthalmia is an indication for an early midface operation, especially combined with significant midface hypoplasia resulting in airway obstruction.

An early airway evaluation by an otolaryngology team is required to understand the full extent of airway issues. Starting from an early age, or at the time of first cranial surgery, an asleep airway evaluation can be done with a diagnostic laryngoscopy and bronchoscopy to assess the entire airway and understand areas of constriction or disease. Constricted nasal airway, laryngomalacia, and tracheocartilagenous rings are commonly observed in these patients and contribute to airway compromise. Early airway interventions, including continuous positive airway pressure and bilevel positive airway pressure therapy, early tonsillectomy, or tracheostomy, should all be considered by the CF team to help optimize and protect the airway. Close follow-up with the otolaryngologist in combination with the other team members ensures proper and safe treatment.

Syndromic patients need to be treated on a case by case basis and a frequent evaluation by the $\mathrm{CF}$ team will allow for optimal care to prevent significant morbidity. The vigilance and level of care for this population is significantly higher than for the single suture, non-syndromic patients. All team members must communicate and work with the coordinator to provide care that is thorough, timely, and beneficial to the patients and their families.

\section{Conclusion}

Craniosynostosis is a complex disorder and the management requires a coordinated effort from a multidisciplinary team. $\mathrm{CF}$ syndromes provide a unique challenge to the $\mathrm{CF}$ team, requiring a constant level of vigilance to meet the needs of these complex issues. Guided by the CF coordinator, both surgical and medical specialists are involved in the care of these patients with the mindset of quality and constantly improving outcomes. A well-coordinated multidisciplinary $\mathrm{CF}$ team is a challenging entity to create, requiring multiple specialists with a common goal who have the passion and perseverance to continually strive to provide better care for their patients.

\section{Disclosure}

The authors report no conflicts of interest in this work.

\section{References}

1. French LR, Jackson IT, Melton LJ 3rd. A population-based study of craniosynostosis. J Clin Epidemiol. 1990;43(1):69-73. 
2. Singer S, Bower C, Southall P, Goldblatt J. Craniosynostosis in western Australia, 1980-1994: a population-based study. Am J Med Genet. 1999;83(5):382-387.

3. Boulet SL, Rasmussen SA, Honein MA. A population-based study of craniosynostosis in metropolitan Atlanta, 1989-2003. Am J Med Genet A. 2008;146A(8):984-991.

4. Kolar JC, Salter EM, Weinberg SM. Preoperative craniofacial dysmorphology in isolated sagittal synostosis: a comprehensive anthropometric evaluation. J Craniofac Surg. 2010;21(5):1404-1410.

5. Lajeunie E, Le Merrer M, Bonaïti-Pellie C, Marchac D, Renier D. Genetic study of scaphocephaly. Am J Med Genet. 1996;62(3):282-285.

6. Fearon JA. Evidence-based medicine: craniosynostosis. Plast Reconstr Surg. 2014;133(5):1261-1275.

7. Rasmussen SA, Olney RS, Holmes LB, Lin AE, Keppler-Noreuil KM, Moore CA; National Birth Defects Prevention Study. Guidelines for case classification for the National Birth Defects Prevention Study. Birth Defects Res A Clin Mol Teratol. 2003;67(3):193-201.
8. Cohen MM Jr. Craniosynostoses: phenotypic/molecular correlations. Am J Med Genet. 1995;56(3):334-339.

9. Lajeunie E, Heuertz S, El Ghouzzi V, et al. Mutation screening in patients with syndromic craniosynostoses indicates that a limited number of recurrent FGFR2 mutations accounts for severe forms of Pfeiffer syndrome. Eur J Hum Genet. 2006;14(3):289-298.

10. Robin NH, Falk MJ, Haldeman-Englert CR. FGFR-related craniosynostosis syndromes. In: Pagon RA, Adam MP, Ardinger HH, et al, editors. GeneReviews ${ }^{\circledR}$ [Internet]. Seattle, WA: University of Washington; 2011.

11. Selber J, Reid RR, Chike-Obi CJ, et al. The changing epidemiologic spectrum of single-suture synostoses. Plast Reconstr Surg. 2008;122(2):527-533.

12. Buchanan EP, Xue AS, Hollier LH Jr. Craniofacial syndromes. Plast Reconstr Surg. 2014;134(1):128e-153e.

13. Bastidas N, Mackay DD, Taylor JA, Bartlett SP. Analysis of the longterm outcomes of nonsyndromic bicoronal synostosis. Plast Reconstr Surg. 2012;130(4):877-883.
Journal of Multidisciplinary Healthcare

\section{Publish your work in this journal}

The Journal of Multidisciplinary Healthcare is an international, peerreviewed open-access journal that aims to represent and publish research in healthcare areas delivered by practitioners of different disciplines. This includes studies and reviews conducted by multidisciplinary teams as well as research which evaluates the results or conduct of such teams or health
Dovepress

care processes in general. The journal covers a very wide range of areas and welcomes submissions from practitioners at all levels, from all over the world. The manuscript management system is completely online and includes a very quick and fair peer-review system. Visit http://www.dovepress.com/ testimonials.php to read real quotes from published authors.

Submit your manuscript here: https://www.dovepress.com/journal-of-multidisciplinary-healthcare-journal 